\title{
Biomechanical and neuromuscular adaptations during the landing phase of a stepping-down task in patients with early or established knee osteoarthritis
}

\author{
Diana C. Sanchez-Ramirez ${ }^{\mathrm{a}}$, Bart Malfait ${ }^{\mathrm{b}}$, Isabel Baert ${ }^{\mathrm{b}, \mathrm{c}, \mathrm{d}}$, Marike van der Leeden ${ }^{\mathrm{e}}$, Jaap van Dieën ${ }^{\mathrm{f}}$, \\ Willem F. Lems ${ }^{\mathrm{g}}$, Joost Dekker ${ }^{\mathrm{h}}$, Frank P. Luyten ${ }^{\mathrm{i}}$, Sabine Verschueren ${ }^{\mathrm{b}, *}$ \\ a Injury Prevention Centre, School of Public Health, University of Alberta, Canada \\ b Musculoskeletal Rehabilitation Research Group, Department of Rehabilitation Sciences, Faculty of Kinesiology and Rehabilitation Sciences, KU Leuven, Belgium \\ c Department of Rehabilitation Sciences and Physiotherapy, Faculty of Medicine and Health Sciences, University of Antwerp, Belgium \\ d Pain in Motion Research Group (www.paininmotion.be), Belgium \\ e Amsterdam Rehabilitation Research Centre Reade, Amsterdam, The Netherlands \\ ${ }^{\mathrm{f}}$ MOVE Research Institute Amsterdam, Faculty of Human Movement Sciences, VU University Amsterdam, The Netherlands \\ ${ }^{g}$ VU University Medical Center, Department of Rheumatology, Amsterdam, The Netherlands \\ ${ }^{\mathrm{h}}$ VU University Medical Center, Department of Rehabilitation Medicine, Amsterdam, The Netherlands \\ i Skeletal Biology \& Engineering Research Center, Faculty of Medicine, KU Leuven, Belgium
}

\section{A R T I C L E I N F O}

\section{Article history:}

Received 15 July 2014

Received in revised form 14 January 2016

Accepted 1 February 2016

\section{Keywords:}

Biomechanics

Stepping-down

Osteoarthritis

Muscle strength

Knee instability

\begin{abstract}
A B S T R A C T
Background: To compare the knee joint kinematics, kinetics and EMG activity patterns during a stepping-down task in patients with knee osteoarthritis (OA) with control subjects.

Methods: 33 women with knee OA (early OA, $n=14$; established OA $n=19$ ) and 14 female control subjects performed a stepping-down task from a $20 \mathrm{~cm}$ step. Knee joint kinematics, kinetics and EMG activity were recorded on the stepping-down leg during the loading phase.

Results: During the stepping-down task patients with established knee OA showed greater normalized medial hamstrings activity $(\mathrm{p}=0.034)$ and greater vastus lateralis-medial hamstrings co-contraction $(\mathrm{p}=0.012)$ than controls. Greater vastus medialis-medial hamstrings co-contraction was found in patients with established OA compared to control subjects $(\mathrm{p}=0.040)$ and to patients with early OA ( $\mathrm{p}=0.023)$. Self-reported knee instability was reported in $7 \%$ and $32 \%$ of the patients with early and established OA, respectively.

Conclusions: The greater EMG co-activity found in established OA might suggest a less efficient use of knee muscles or an attempt to compensate for greater knee laxity usually present in patients with established OA. In the early stage of the disease, the biomechanical and neuromuscular control of stepping-down is not altered compared to healthy controls.
\end{abstract}

(c) 2016 Elsevier B.V. All rights reserved.

\section{Introduction}

Osteoarthritis (OA) is a highly prevalent joint disease [1], which has been counted globally as the sixth leading cause of moderate-to-severe disability and the eight cause of burden disease in the European region [2]. Patients with OA commonly experience pain, stiffness, reduction in the range of motion and muscle weakness, factors associated with activity limitations such as the difficulty to stand up from a chair, walk or climb stairs [3,4]. Studies carried out in patients with OA have documented the use of compensatory strategies during gait such as decreased walking speed [5], decreased cadence [6], decreased stride

\footnotetext{
* Corresponding author at: Katholieke Universiteit Leuven, Tervuursevest 101, 3001 Leuven, Belgium.

E-mail address: sabine.verschueren@faber.kuleuven.be (S. Verschueren).
}

length [7], decreased knee flexion angle during the loading response phase [8], increased step width [9], increased hip internal rotation and increased lateral trunk lean [9]. Modifications in knee loading distribution such as increases in knee adduction moment (KAM) and knee adduction angular impulse have also been reported [10,11]. A direct association between higher KAM and severity of knee AO has been found $[10,11]$.

Changes in electromyography (EMG) activity patterns during gait including increased activity of hamstrings and increased cocontraction have been documented [12]. This increased co-activation might be an adaptation of the individual with $\mathrm{OA}$ to deal with pain and instability generated by the loss of joint integrity. In this view, this co-activation could increase the stiffness of the joint promoting knee stability [9]. On the other hand, those gait modifications and increased co-activation could interfere with the distribution of the load on the knee joint, leading to further joint damage and disease progression [8]. 
The kinematic and kinetic characteristics during gait and stair climbing have been extensively studied in patients with knee $\mathrm{OA}$ in comparison with healthy subjects $[8,13,14]$. However, analysis of the biomechanical characteristics involved in other activities of daily living like stepping-down from a sidewalk still need to be further analysed, as stepping-down is a task that elicits complaints of instability and pain [15-17]. In addition some studies have differentiated between the characteristics of patients in different stages of the disease (early vs. established OA) but they often did not use MRI to define their groups. Knowledge of the stage in the process in which modifications in movement patterns occur might be helpful in the understanding of disease development and/or progression. It is possible that patients at risk or with early $\mathrm{OA}$, defined as joint pain with structural damage detected on MRI but hardly visible on X-rays [18], respond better to certain interventions than patients with established $\mathrm{OA}$.

Patients with knee OA often complain of knee instability, defined as the sensation of buckling, shifting or giving way, which usually translates into activity limitations [19]. Previous studies have estimated that between $12 \%$ and $65 \%$ of this group of patients have reported at least one episode of knee instability during the past three months [20,21].

Knee joint stabilization is thought to be influenced by active muscle force contraction and passive ligaments restraints, both of which are usually affected in patients with knee OA [20,22,23]. Evidence has shown an association between self-reported knee instability and isokinetic average knee muscle weakness [21], but not with passive knee laxity in this group of patients [24]. However, failure to control the knee usually occurs during dynamic activities [19]. Therefore, in an attempt to further explore knee stability in patients with $\mathrm{OA}$, recent studies have aimed to identify the objective biomechanical and/or neuromuscular performance characteristics associated with knee instability. Those studies have reported an association between greater knee adduction moment and medial knee laxity during gait [10], and lower medial knee muscle co-contraction prior to platform perturbations in patients with medial compartment knee OA [25]. Nevertheless, to the best of our knowledge the biomechanical and neuromuscular components associated with the sensation of knee instability in those patients have not been fully recognized. In addition, further study of knee instability in patients with early OA might help to clarify the association between knee instability and disease severity. In knee OA, disease progression leads to a structural deterioration which subsequently can cause joint instability, as often mentioned in OA. Nevertheless, joint instability can also contribute to further disease progression [26].

During stair descent loading forces across the knee joints are higher than during stair ascent and level walking, making it a more challenging task requiring good neuromuscular control to obtain good shock absorption and knee stability $[27,28]$. Particularly the early stance phase is important during which the ground reaction forces need to be attenuated (by eccentric muscle activity) as weight is loaded onto one limb [27]. Therefore, the stance phase of a step-down task was assessed in the present study to represent the stance phase of stair descent. The stepping-down task has been used successfully to study movement strategies in elderly subjects [29] and dynamic knee instability in a patient with anterior cruciate ligament deficiency $[16,30]$. Therefore, the purpose of this study was to investigate the joint kinematics, kinetics and EMG activity patterns in patients with early or established $\mathrm{OA}$ of the knee during a stepping-down task.

We hypothesise that the analysis of knee kinematics, kinetic and EMG activity during the performance of the stepping-down task might elucidate relevant biomechanical characteristics associated with compensatory strategies for instability or pain used by patients with knee OA (early and established). Secondarily, this task might help to explore biomechanical and neuromuscular strategies associated with self-reported knee instability in this group of patients. The results might contribute to the design of intervention strategies directed to treat difficulties of mobility and knee instability in patients with knee OA.

\section{Methods}

\subsection{Subjects}

A convenience sample of 47 females was included in this study (Table 1$)$. Patients with OA $(n=33)$ were recruited by a rheumatologist or orthopaedic surgeon from the University Hospitals Leuven. Fourteen patients were classified as early OA based on a combination of pain, Kellgren/Lawrance (KL) score $=0$ or 1 on radiography and presence of at least two of four MRI criteria: (1) $\geq$ BLOCKS grade 2 for size cartilage loss, (2) $\geq$ BLOCKS for percentage full-thickness cartilage loss, (3) signs of meniscal degeneration, and (4) $\geq$ BLOCKS for size of BMLs in any compartment [18]. Nineteen patients were classified as unilateral or bilateral established knee OA based on the criteria from the American College of Rheumatology (ACR) [31] and $\mathrm{KL} \geq 2 \pm[32,33]$. Control subjects $(n=14)$ with no history of knee symptoms or characteristics associated with knee $\mathrm{OA}$ and $\mathrm{KL}=0$ were recruited from cultural or social organizations. Demographic, clinical, radiographic, neuromuscular and biomechanical factors related to OA were assessed. Total knee replacement, rheumatoid arthritis or any other form of inflammatory arthritis (i.e. crystal arthropathy, septic arthritis, spondylarthropathy) were considered exclusion criteria. All the participants provided written inform consent before testing. The study was approved by the local Ethics Committee.

\subsection{Measures}

\subsubsection{Loading phase of stepping-down task}

The subjects were instructed to step down from a wooden step $(20 \mathrm{~cm}$ ) (Figure 1) onto a force plate with the evaluated limb and to step forward with the other limb. Subjects ended in quiet stance on both legs in front of the force plate (Figure 2). The arms were kept flexed across the chest to avoid obstruction of the visibility of the reflective markers. All patients wore standard sport shoes (kelme indoor copa). A task cycle was considered from the first contact with the force plate (touch-down) until the toe-off from the force plate with the evaluated limb. In a single session, three trials per patient were recorded. Both limbs were assessed but only the index leg (see statistical analysis) was included in the analysis.

\subsubsection{Knee instability}

Self-reported knee instability was evaluated based on a questionnaire from Felson et al. $[19,20]$ in which a sensation of knee buckling, shifting or giving away during the past three months was inquired. Persons reporting knee instability were additionally asked for the number of episodes of instability, on which leg it was experienced. Knee instability was dichotomized as "0" if they did not report episodes and " 1 " if they reported episodes of instability during the past three months [18]. An additional question about history of knee injury ("Did you ever have a knee injury?" yes/no) was formulated to persons who reported to have had at least one episode of knee instability, this with the intention to explore whether the sensation of instability could be due to another cause such as traumatic injury.

\subsubsection{Muscle strength}

Knee muscle strength was assessed using the Biodex System 3 Pro (Biodex Medical System, Shirley, NY, USA). An initial practise attempt was used for the participants to become familiar with the movements required. The patients performed three maximal test repetitions to measure the isokinetic strength of the knee extensor muscles (mainly quadriceps) and knee flexor muscles (mainly hamstrings) for each knee, at $60^{\circ} / \mathrm{s}$." [34]. Isometric knee extension and flexion were measured in $60^{\circ}$ flexion position. The peaks of three trials were averaged in each leg separately for isometric and isokinetic assessments (quadriceps and hamstrings torques $(\mathrm{Nm})$ ) and divided by patient's weight 
Table 1

Characteristics of study group.

\begin{tabular}{|c|c|c|c|c|c|c|c|}
\hline & \multirow{2}{*}{$\begin{array}{l}\text { Control subjects } \\
(\mathrm{n}=14)\end{array}$} & \multirow{2}{*}{$\begin{array}{l}\text { Early knee } \mathrm{OA} \\
(\mathrm{n}=14)\end{array}$} & \multirow{2}{*}{$\begin{array}{l}\text { Established knee OA } \\
(\mathrm{n}=19)\end{array}$} & \multirow[t]{2}{*}{$\mathrm{p}$-Value } & \multicolumn{3}{|c|}{ Post hoc p-value } \\
\hline & & & & & $\begin{array}{l}\text { Established } \\
\text { vs } \\
\text { control }\end{array}$ & $\begin{array}{l}\text { Early } \\
\text { vs } \\
\text { control }\end{array}$ & $\begin{array}{l}\text { Established } \\
\text { vs } \\
\text { early }\end{array}$ \\
\hline Age, in years & $68.0 \pm 3.9$ & $70.4 \pm 4.6$ & $68.37 \pm 6.7$ & 0.457 & & & \\
\hline Height, m & $1.63 \pm 0.1$ & $1.63 \pm 0.1$ & $1.59 \pm 0.1$ & 0.080 & & & \\
\hline Weight, kg & $69.9 \pm 9.3$ & $73.6 \pm 10.3$ & $72.1 \pm 10.4$ & 0.621 & & & \\
\hline Body mass index, $\mathrm{kg} / \mathrm{m}^{2}$ & $26.2 \pm 2.9$ & $27.8 \pm 4.7$ & $28.5 \pm 4.6$ & 0.290 & & & \\
\hline 1 & - & $14(100)$ & - & & & & \\
\hline$\geq 2 \pm$ & - & - & $19(100)$ & & & & \\
\hline \multicolumn{8}{|l|}{ Clinical characteristics } \\
\hline VAS knee pain $(0-10)$ & $0.86 \pm 1.3$ & $1.64 \pm 2.2$ & $2.95 \pm 2.7$ & $0.033^{*}$ & $0.029^{*}$ & 0.624 & 0.233 \\
\hline KOOS pain score $(0-100)$ & $91.24 \pm 8.4$ & $82.71 \pm 15.7$ & $80.36 \pm 14.8$ & 0.078 & & & \\
\hline KOOS symptoms score $(0-100)$ & $89.50 \pm 10.1$ & $78.77 \pm 15.4$ & $74.96 \pm 18.2$ & $0.033^{*}$ & $0.028^{*}$ & 0.163 & 0.764 \\
\hline Self-reported knee instability, n (\%) & $0(0)$ & $1(7)$ & $6(32)$ & $0.026^{*}$ & $0.020^{*}$ & 0.309 & 0.090 \\
\hline Missing, $\mathrm{n}(\%)$ & $0(0)$ & $0(0)$ & $1(6)$ & & & & \\
\hline \multicolumn{8}{|l|}{ Muscle strength } \\
\hline \multicolumn{8}{|l|}{ Isokinetic $60^{\circ} / \mathrm{s}$} \\
\hline Average knee muscle strength $(\mathrm{Nm} / \mathrm{kg})^{\mathrm{a}}$ & $1.00 \pm 0.2$ & $0.88 \pm 0.2$ & $0.91 \pm 0.2$ & 0.242 & & & \\
\hline Extensor muscle strength $(\mathrm{Nm} / \mathrm{kg})$ & $1.20 \pm 0.3$ & $1.01 \pm 0.2$ & $1.08 \pm 0.3$ & 0.227 & & & \\
\hline Flexor muscle strength $(\mathrm{Nm} / \mathrm{kg})$ & $0.81 \pm 0.2$ & $0.76 \pm 0.2$ & $0.68 \pm 0.2$ & 0.085 & & & \\
\hline \multicolumn{8}{|l|}{ Isometric $60^{\circ}$} \\
\hline Average knee muscle strength $(\mathrm{Nm} / \mathrm{kg})^{\mathrm{a}}$ & $1.10 \pm 0.2$ & $0.99 \pm 0.2$ & $0.95 \pm 0.1$ & 0.078 & & & \\
\hline Extensor muscle strength $(\mathrm{Nm} / \mathrm{kg})$ & $1.43 \pm 0.4$ & $1.28 \pm 0.2$ & $1.30 \pm 0.3$ & 0.362 & & & \\
\hline Flexor muscle strength $(\mathrm{Nm} / \mathrm{kg})$ & $0.76 \pm 0.1$ & $0.68 \pm 0.2$ & $0.62 \pm 0.1$ & $0.015^{*}$ & $0.011^{*}$ & 0.217 & 0.445 \\
\hline \multicolumn{8}{|l|}{ Activity limitations } \\
\hline KOOS ADL score (0-100) & $94.21 \pm 6.5$ & $83.56 \pm 13.2$ & $83.78 \pm 14.7$ & $0.037^{*}$ & 0.058 & 0.066 & 0.999 \\
\hline Stair climbing test, seconds & $5.67 \pm 1.1$ & $5.50 \pm 1.1$ & $5.94 \pm 1.0$ & 0.510 & & & \\
\hline Get up and go test, seconds & $6.53 \pm 1.7$ & $6.28 \pm 1.4$ & $7.09 \pm 1.6$ & 0.338 & & & \\
\hline
\end{tabular}

Mean \pm standard deviation (SD), unless other stated. OA = osteoarthritis; K/L = Kellgren/Lawrence; VAS = visual analogue scale; KOOS = Knee Injury and Osteoarthritis Outcome Score. Bold $\chi^{2}$.

${ }^{a}$ Average knee extensor and flexor muscle strength.

b Time from touch-down to toe-off.

* $\mathrm{p} \leq 0.05$ significant difference between groups.

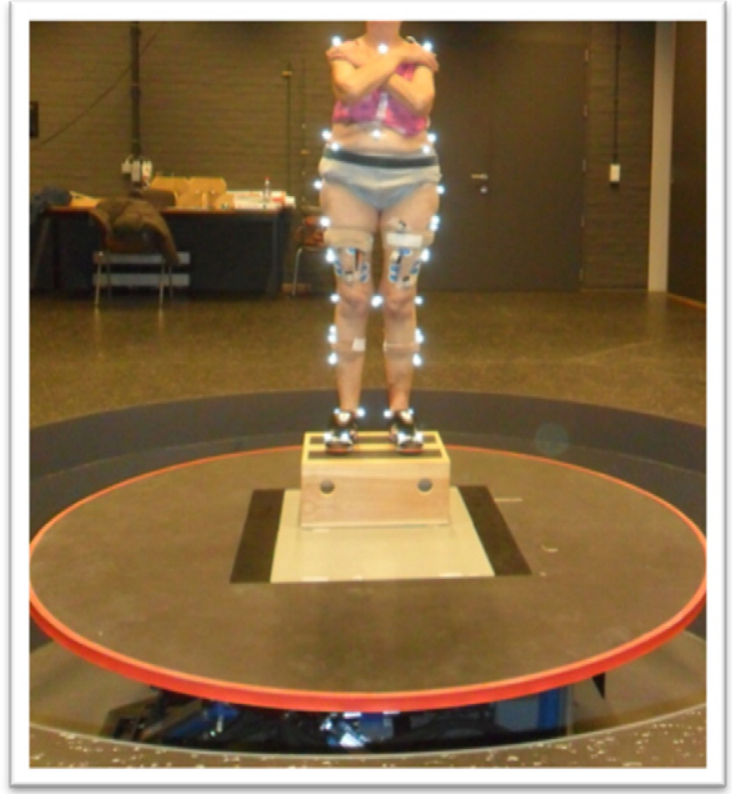

Figure 1. Subject in the initial position.
( $\mathrm{kg}$ ). This measure (in $\mathrm{Nm} / \mathrm{kg}$ ) has excellent intra-rater reliability (ICC 0.93 ) in knee OA patients [35,36].

\subsubsection{Knee joint alignment}

Knee alignment was measured from anterior-posterior weight bearing radiograph of the lower limbs (Oldelft, Triahlon, Afga ADC M Compact Plus) by a single experienced rheumatologist (FL). The alignment of the mechanical axis was reported as varus if $\leq-3^{\circ}$ or valgus if $\geq 3^{\circ}$. Knee alignment between $-3^{\circ}$ and three degrees was classified as neutral $[37,38]$.

\subsubsection{Activity limitation}

Activity limitations were assessed subjectively using the Dutch version of the Knee Injury and Osteoarthritis Outcome Score (KOOS) [39] which ranges from 0 (poor outcome) to 100 (good outcome), and objectively using the stair test and the get up and go test (GUG). In the stair test [35], subjects were instructed to climb five stair steps $(15 \mathrm{~cm}$ high), turn around and descend the stairs. Participants were encouraged not to use the handrail, but were not prohibited from doing so for safety. In the GUG test [35], subjects were sitting on a high standard chair $(49 \mathrm{~cm})$, they were told to stand up without help of the arms on the command "go", and walk three metres through an unobstructed corridor as fast as possible, without running. Once they reached a mark on the floor, the subjects turned around, returned to the chair and sat down. Patients who normally used walking devices were allowed to use them during the test. All subjects were wearing standard sport 

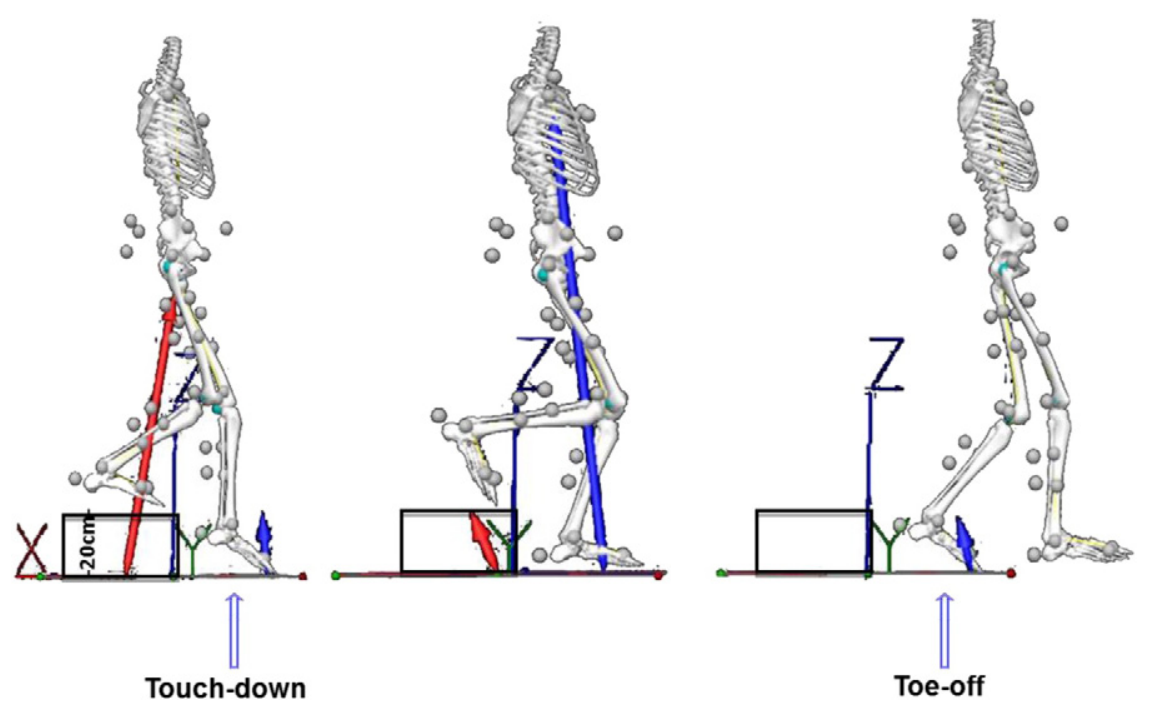

Figure 2. Stepping-down task.

shoes during the performance of the tests. The time in seconds was recorded for both tests; longer time was considered a higher activity limitation. For each test, the mean value of three trials was calculated. Both tests have shown good reliability and validity [35].

\subsubsection{Pain and symptoms}

Pain was assessed with the visual analogue scale (VAS), the patient was asked to range the sensation of pain during the last week from 0 (none) to 10 (severe pain). The Dutch version of the KOOS questionnaire was also used to assess pain and general symptoms, ranging them from 0 (poor outcome) to 100 (good outcome) [39].

\subsection{Data capture}

The stepping-down task was tracked using 6 MX-T20 optoelectronic cameras (Vicon, Oxford Metrics, UK) collected at $100 \mathrm{~Hz}$ in Nexus (Vicon). Eight body segments (trunk, pelvis, upper-lower legs and feet) were identified by 46 spherical reflective markers of $14 \mathrm{~mm}$ diameter (see Supplemental Digital Content from Malfait et al. [40] available at http://links.lww.com/MSS/A369). Segmental coordinate systems were identified as reported previously $[41,42]$. Simultaneously (time synchronized), data from the force plate (AMTI Watertown, MA, USA) and the EMG were sampled at $1500 \mathrm{~Hz}$ [34].

EMG activity of the vastus medialis (VM), vastus lateralis (VL), medial hamstrings $(\mathrm{MH})$ and lateral hamstrings ( $\mathrm{LH}$ ) was recorded bilaterally using a 16-channel system wireless surface EMG system (Aurion, Italy) and silver-silver chloride, pre-gelled bipolar surface EMG electrodes (Ambu Blue Sensor, Ballerup, Danmark). The electrodes were placed over the muscle belly two centimetres centre to centre in line with the muscle fibres, and with an inter-electrode distance of three centimetres to reduce the possibility of cross-talk between neighbouring muscles [43]. Isolated manual muscle test [44] was used to validate the placement of the electrodes and to assess for cross talk [45]. Skin surface was previously shaved and cleaned with 70\% isopropyl alcohol to reduce impedance.

\subsection{Data processing and analysis}

Separate trials were used for anatomical calibration and for calculation of hip and knee joint centres and functional axis of the model [41, 46,47]. Marker trajectories and force plate data were both filtered using a 4th order low pass Butterworth filter with a cut off frequency of $20 \mathrm{~Hz}$, based on previous studies [40,48]. Touch-down and toe-off events were defined based on the vertical force crossing a $20 \mathrm{~N}$ threshold. Joint knee flexion angles were calculated at touch-down and at the point of peak knee flexion during the task (peak knee flexion angle $($ PKFA)) (Figure 3). Knee adduction moment, defined as the external load applied at the joint moving the tibia to varus position was calculated using inverse dynamics and normalized to body mass $(\mathrm{Nm} / \mathrm{kg})$. There were no clearly defined early and late peak adduction moments during the performance of the stepping-down task. Therefore, the peak knee adduction moment (PKAM) as well as the integral of the knee adduction angular impulse (KAAI) over the complete stance phase $(\mathrm{Nms} / \mathrm{kg})$ were included in the analyses. The average of three stepping-down trials was calculated for all biomechanical parameters for each participant [49]. All modelling and analyses were undertaken in Visual 3D (v.4.83, C-motion, Germantown, MD, USA) using geometric volumes to represent segments based on cadaver segmental data as described in previous studies [40,50].

EMG signals were high pass filtered at a cut-off frequency of $10 \mathrm{~Hz}$ [51]. The rectified EMG signals were also filtered with a 4th order zero-lag low pass Butterworth filter at a cut-off frequency of $50 \mathrm{~Hz}$ and subsequently normalized to the peak EMG activity of each muscle during the stepping-down task cycle [52,53]. The root mean square (RMS) from touch-down to the PKFA was calculated for each muscle on the stepping-down leg.

Muscle co-contraction index (CCI) for the medial (VMMH = vastus medialis-medial hamstrings) and lateral (VLLH = vastus lateralislateral hamstrings) sides of the knee joint, as well as for the oblique surface of the knee joint (VLMH = vastus lateralis-medial hamstrings), were calculated from touch-down to the PKFA according to the following equation [54]:

$$
C C I=E M G S / E M G L \times(E M G S+E M G L)
$$

in which EMGS is the normalized magnitude of the EMG signal for the less active muscle and EMGL is the normalized magnitude of the EMG signal for the most active muscle. To determine whether medial to lateral co-contraction was imbalanced muscle co-contraction medial to lateral ratio was calculated dividing medial co-contraction index with lateral co-contraction index [55].

The co-contraction index used here represents the balance of EMG activity between pairs of antagonistic muscles and it is commonly used in the literature [55]. However, it is important to consider that EMG signal does not reflect muscle force and hence this index does not provide direct information about the magnitude of knee loading. 


\section{Knee flexion angle}

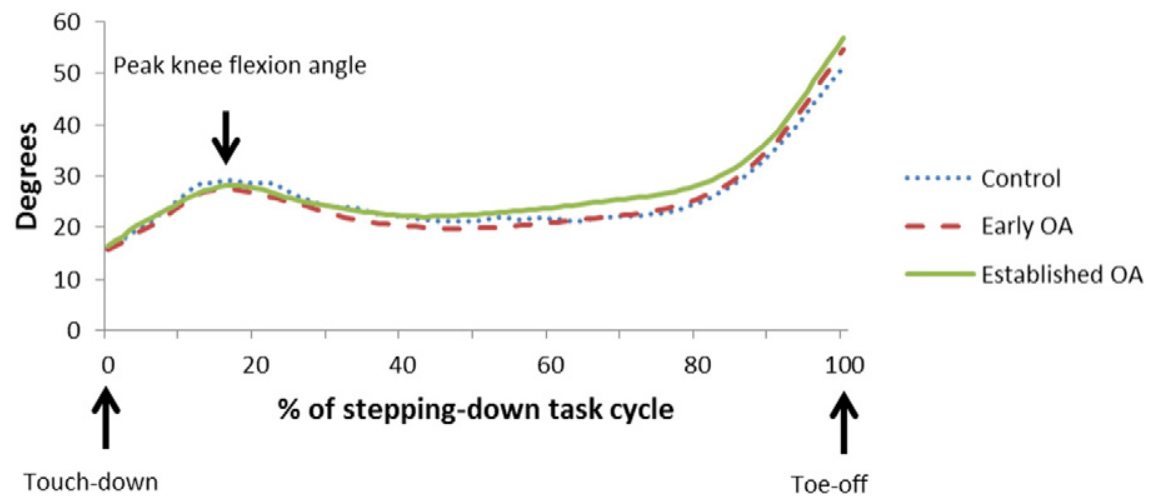

Figure 3. Lines represent the mean knee flexion angle per group.

\subsection{Statistical analysis}

For the patients with knee $\mathrm{OA}$ an index knee was selected using the following decision tree: 1) knee with established or early OA (ACR and KL score), if OA diagnosis was the same in both knees, 2) instable knee and 3) painful knee. In participants in whom an index knee could not be defined based on these signs, a random index joint was assigned. For the control subjects the right knee was used as reference. The variables related to the index knee were used in the analyses.

Descriptive statistics were used to characterize the study population, as well as the patients with knee OA and control subjects separately. Percentages were used for categorical variables, and means and SDs for continuous variables. One-way analyses of variance (ANOVA) and chi-square tests were used to analyse the differences in the distribution of the variables between the three subgroups.

ANOVA-Tukey post hoc tests were used to test the group difference in knee joint angles, external moments and EMG activity between subjects with established OA, early OA and control subjects. Chi square tests $\left(\chi^{2}\right)$ were used to compare self-reported knee instability between the study groups. Independent t-tests were used to compare the patients' characteristics, joint kinematics, kinetics and EMG activity patterns during a stepping-down task in patients with and without self-reported knee instability. Statistical significance was accepted at pvalues $\leq 0.05$. All analyses were performed using SPSS software, version 17.0 (SPSS, Chicago, IL).

\section{Results}

3.1. Descriptives

The mean age of the females that participated in the study was $68.9( \pm 5.4)$ years old. Patient with established knee OA had significantly more knee pain $(\mathrm{p}=0.029)$ and lower isometric knee flexor muscle strength than the control group $(p=0.011)$. A lower percentage of patients with established OA had their knees in neutral alignment compared with patients with early $\mathrm{OA}(\mathrm{p}=0.017)$, the difference was borderline significant when comparing with control subjects $(\mathrm{p}=0.051)$. No significant group differences were found in other variables assessed including activity limitations. Further, demographic, clinical and neuromuscular characteristics are shown in the Table 1

3.1.1. Knee biomechanics and EMG activity patterns during the loading phase of steppingdown task

There were no significant differences in kinematics or kinetics between the groups with knee OA (early-established) and/or the control group during the loading phase of the stepping-down task. Patients with established knee OA showed greater normalized medial hamstrings activity $(\mathrm{p}=0.034)$ and greater vastus lateralis-medial hamstrings co-contraction ( $p=0.012$ ) compared with the control subjects. Higher vastus medialismedial hamstrings co-contraction was found in patients with established OA compared

Table 2

Kinematics, kinetics and muscle activity during the stepping-down task.

\begin{tabular}{|c|c|c|c|c|c|c|c|}
\hline & \multirow{2}{*}{$\begin{array}{l}\text { Control } \\
(n=14)\end{array}$} & \multirow{2}{*}{$\begin{array}{l}\text { Early OA } \\
(n=14)\end{array}$} & \multirow{2}{*}{$\begin{array}{l}\text { Established } \\
\text { OA } \\
(n=19)\end{array}$} & \multirow[t]{2}{*}{ p-Value } & \multicolumn{3}{|c|}{ Post hoc p-value } \\
\hline & & & & & $\begin{array}{l}\text { Established vs } \\
\text { control }\end{array}$ & Early vs control & $\begin{array}{l}\text { Established vs } \\
\text { early }\end{array}$ \\
\hline \multicolumn{8}{|l|}{ Kinematics and kinetics } \\
\hline Knee flexion angle at touch-down, degrees & $16.01 \pm 3.0$ & $15.66 \pm 3.5$ & $16.78 \pm 4.0$ & 0.651 & & & \\
\hline Peak knee flexion angle (PKFA), degrees & $31.01 \pm 6.1$ & $29.02 \pm 4.2$ & $30.59 \pm 6.7$ & 0.636 & & & \\
\hline Knee flexion excursion, degrees & $15.00 \pm 4.4$ & $13.36 \pm 3.8$ & $13.81 \pm 4.3$ & 0.568 & & & \\
\hline Peak knee adduction moment (PKAM), Nm/kg & $0.37 \pm 0.4$ & $0.29 \pm 0.1$ & $0.30 \pm 0.3$ & 0.697 & & & \\
\hline Knee adduction angular impulse moment (KAAI), Nms/kg & $0.23 \pm 0.3$ & $0.16 \pm 0.1$ & $0.17 \pm 0.2$ & 0.598 & & & \\
\hline Peak knee flexion moment, $\mathrm{Nm} / \mathrm{kg}$ & $-0.68 \pm 0.4$ & $-0.65 \pm 0.1$ & $-0.69 \pm 0.3$ & 0.908 & & & \\
\hline Peak knee external rotation moment, $\mathrm{Nm} / \mathrm{kg}$ & $-0.07 \pm 0.1$ & $-0.07 \pm 0.1$ & $-0.10 \pm 0.1$ & 0.617 & & & \\
\hline \multicolumn{8}{|l|}{ Muscle activity $^{\mathrm{a}}$} \\
\hline Vastus medialis (VM) & $0.42 \pm 0.1$ & $0.41 \pm 0.1$ & $0.43 \pm 0.1$ & 0.720 & & & \\
\hline Vastus lateralis (VL) & $0.44 \pm 0.1$ & $0.43 \pm 0.1$ & $0.43 \pm 0.1$ & 0.902 & & & \\
\hline Medialis hamstrings ( $\mathrm{MH})$ & $0.29 \pm 0.1$ & $0.30 \pm 0.1$ & $0.37 \pm 0.1$ & $0.025^{*}$ & $0.034^{*}$ & 0.909 & 0.093 \\
\hline Lateral hamstrings ( $\mathrm{LH})$ & $0.33 \pm 0.1$ & $0.36 \pm 0.1$ & $0.39 \pm 0.1$ & 0.298 & & & \\
\hline VMMH co-contraction & $0.50 \pm 0.2$ & $0.48 \pm 0.1$ & $0.64 \pm 0.2$ & $0.012^{*}$ & $0.040^{*}$ & 0.976 & $0.023^{*}$ \\
\hline VLLH co-contraction & $0.55 \pm 0.2$ & $0.64 \pm 0.2$ & $0.65 \pm 0.2$ & 0.310 & & & \\
\hline VLMH co-contraction & $0.47 \pm 0.2$ & $0.51 \pm 0.2$ & $0.64 \pm 0.2$ & $0.009^{*}$ & $0.012^{*}$ & 0.791 & 0.064 \\
\hline VMMH/VLLH co-contraction ratio & $0.98 \pm 0.3$ & $0.89 \pm 0.5$ & $1.04 \pm 0.4$ & 0.605 & & & \\
\hline
\end{tabular}

Mean \pm standard deviation (SD).

a Root mean square from touch-down to PKFA during the stepping-down task.

* $\mathrm{p} \leq 0.05$ significant difference between groups. 
with control subjects $(p=0.040)$ and to patients with early OA ( $p=0.023)$ (Table 2$)$ (Figure 4).

\subsection{Self-reported knee instability}

Seven patients ( $15 \%$ ) with knee $O A$ (early $n=1$; established $n=6$ ) reported to have at least one episode of knee instability during the past three months. The incidence of instability was significantly higher in the group with established OA compared with the control $(p=0.020)$. Neither of the patients with self-reported knee instability reported a previous knee injury. None of the characteristics studied such as the biomechanics and EMG activity patterns during the performance of the loading phase of stepping-down task (Table 3) were significantly different between patients with or without self- reported knee instability. However, patients with self-reported knee instability showed significantly lower knee muscle strength compared with subjects without self-reported knee instability (Figure 5).

\section{Discussion}

This study investigated the biomechanical and neuromuscular strategies during the loading phase of a stepping-down task in a group of patients with early or established knee OA compared to a healthy control group. The main study results showed no difference in the kinematic or

\section{Muscle co-contraction during the stepping-down task}

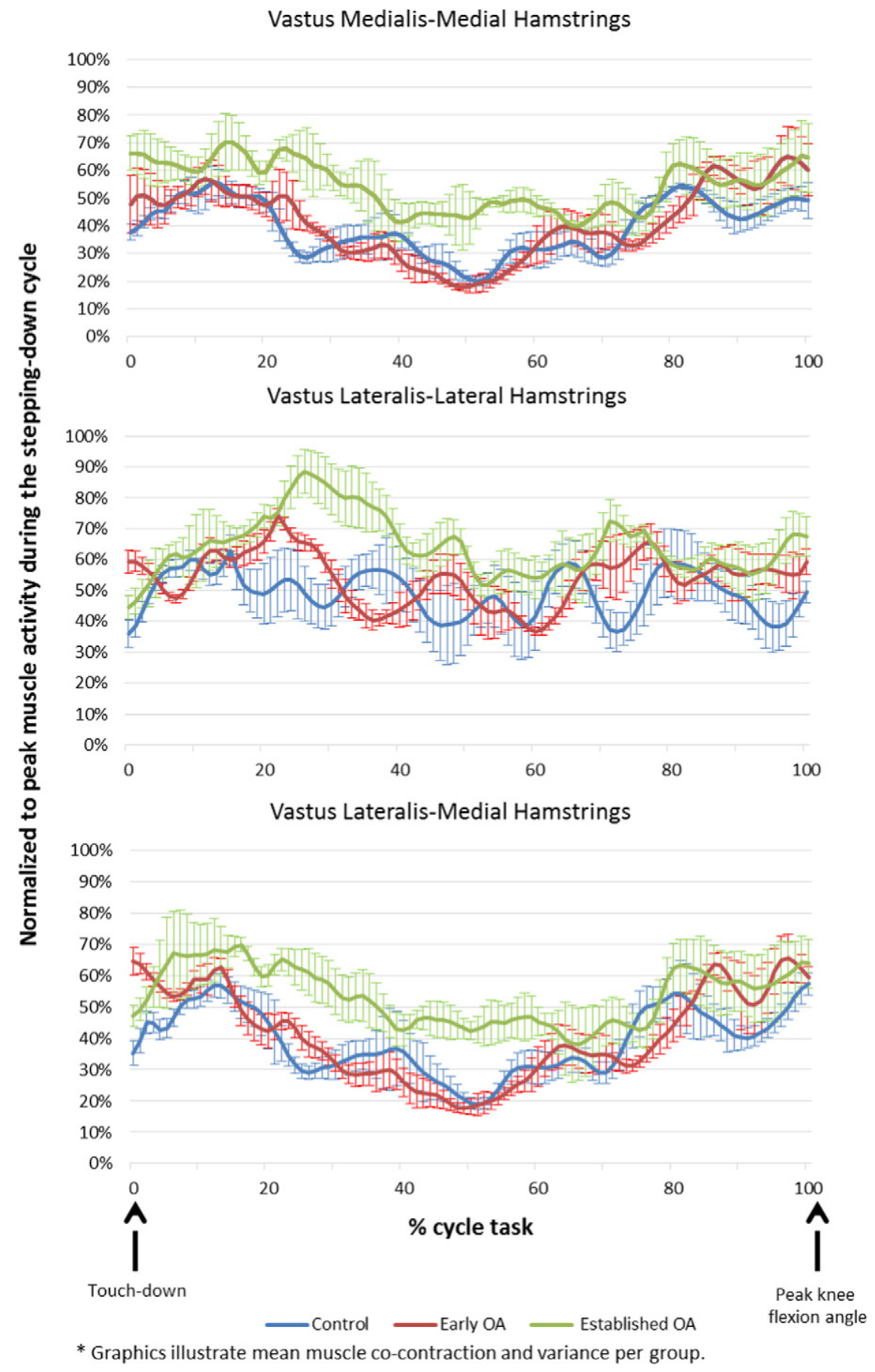

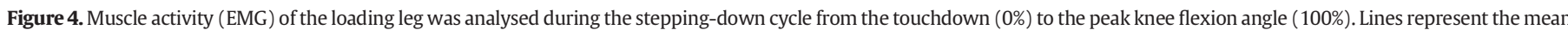
muscle co-contraction and variance per group. 
kinetic characteristics during the loading phase of a stepping-down task between the three groups. However, greater muscle (co-)contraction patterns were observed in patients with established knee OA compared with control subjects and patients with early $\mathrm{OA}$.

There were no significant differences in sagittal plane kinematics or kinetics during the loading phase of the stepping-down task between patients with early or established OA, and control subjects. Based on these results, it is possible to conclude that an isolated stepping-down task might not be challenging enough to identify kinematic and kinetic differences between the three groups studied. Decreased knee flexion angle excursion was previously reported in patients with established knee OA during a step down task from $20 \mathrm{~cm}$ [8]. However, in the present study, no significant difference in the knee flexion angle at touchdown, at peak knee flexion during the stance phase or in flexion excursion was found between the three groups studied during the loading phase of the stepping-down task. The difference between both studies could be related to the fact that in the study carried out by Childs et al. [8] the subjects continued to walk forward several steps after stepping-down, which may have allowed a more natural performance of the task. The setting in our laboratory restricted the task only to one step forward after stepping down (Figure 1).

Peak knee adduction moments and knee adduction angular impulse during the loading phase of the stepping-down task were not significantly different between the three groups (early OA, established OA and control subjects). Higher adduction moments during gait have been previously found in patients with established knee OA in the medial compartment $[10,11,56]$, however not in subjects with early OA. It is therefore expected that knee OA severity in the medialcompartment is associated with greater peak adduction moments. However, the discrepancy with the results from the present study might be explained by the more heterogeneous distribution of the structural features in the knee joint which is in line with previous findings from Messier's et al. [57]. In addition, it is possible that the lack of association between OA severity and knee adduction moment found during gait by other authors might not be present during the loading phase of the stepping-down task evaluated in the present study. Assessing the kinematics and kinetics of the supporting leg, in the step descent phase might reveal more differences.

Greater medial hamstrings $(\mathrm{MH})$ activity was exhibited in patients with established knee OA compared with control subjects. Additionally, greater medial muscle co-contraction (VMMH) was found in patient with established OA compared to control subjects and to patients with early knee OA. These are in accordance with previous findings and may reflect an effort to compensate higher medial knee laxity, usually present during gait in patients with established OA [23-25]. Additionally, greater co-contraction of the posterior-medial $(\mathrm{MH})$ and the lateralanterior $(\mathrm{VL})$ sides of the knee was found in the group of patients with established knee OA compared with the control subjects. According to Rudolph et al. [54] high-level co-contraction of opposing muscle groups could result in higher joint compression. These findings suggest not only a higher medial compression of the medial knee compartment of the knee, but also an overall increase in the compressive load through the knee surface in patients with established OA. Previous evidence have suggested that an increase in muscle co-contraction may lead to an increase of the cumulative load on the knee, which in turn might translate in further knee joint damage and disease progression [8].

Seven patients with knee OA without a known history of knee injury reported to have at least one episode of knee instability during the past three months. However, none of the participants reported to have a feeling of knee instability during the performance of the steppingdown task in our laboratory. In the present study, incidence of instability seems to increase with the severity of the disease. However, to the best of our knowledge there is no published evidence to prove this finding and the sample of patients with knee instability in this study was too small to draw firm conclusion. It is possible that the study of biomechanical characteristics of subjects with self-reported knee instability during the stepping-down task might be useful to objectively identify performance characteristics associated with knee instability, which could contribute to develop appropriate strategies oriented to counteract instability in those patients. Nevertheless, probably due to the small number of patients with self-reported knee instability within this study group, the results of this study did not support our hypothesis. Therefore, studies in a larger sample population with self-reported knee instability during the performance of a more challenging task might be needed to further clarify whether or not biomechanical and neuromuscular performance based characteristics might be associated with the feeling of instability in patients with knee OA.

In patients with established knee OA showing muscle weakness, muscle strength training (both extensor and flexor knee muscles [34]) as well as neuromuscular training leading to a selective EMG activity instead of increased and prolonged co-contraction patterns $[58,59]$ may be recommended to preserve joint integrity (Hodges et al. 2015). The influence of neuromuscular training on knee stability still needs to be elucidated. Further studies are needed to disentangle which of the biomechanical and neuromuscular performance based characteristics are driven by pain, instability, structural changes and/or other factors. Overall, it appears necessary to optimize the rehabilitation strategies directed to decrease an abnormal joint loading during diverse activities of

Table 3

Kinematics, kinetics and muscle activity during the stepping-down task in patients with knee OA ( $\mathrm{n}=33$ ) with or without self-reported knee instability.

\begin{tabular}{|c|c|c|c|c|c|}
\hline & \multicolumn{5}{|c|}{ Self-reported knee instability } \\
\hline & Yes $(n=7)$ & No $(n=26)$ & p-Value & $r$ & p-Value \\
\hline \multicolumn{6}{|l|}{ Kinematics and kinetics } \\
\hline Knee flexion angle at touch-down, degrees & $15.12 \pm 5.4$ & $16.62 \pm 3.2$ & 0.355 & -0.166 & 0.355 \\
\hline Peak knee flexion angle, degrees & $30.09 \pm 8.2$ & $29.88 \pm 5.1$ & 0.951 & 0.015 & 0.934 \\
\hline Knee flexion excursion, degrees & $14.97 \pm 3.5$ & $13.26 \pm 4.2$ & 0.331 & 0.174 & 0.331 \\
\hline Peak knee adduction moment (PKAM), Nm/kg & $0.28 \pm 0.4$ & $0.30 \pm 0.2$ & 0.874 & -0.029 & 0.874 \\
\hline Knee adduction angular impulse moment (KAAI), Nms/kg & $0.22 \pm 0.1$ & $0.15 \pm 0.2$ & 0.249 & 0.206 & 0.249 \\
\hline Peak knee flexion moment, $\mathrm{Nm} / \mathrm{kg}$ & $-0.73 \pm 0.4$ & $-0.65 \pm 0.2$ & 0.659 & -0.125 & 0.490 \\
\hline Peak knee external rotation moment, $\mathrm{Nm} / \mathrm{kg}$ & $-0.11 \pm 0.2$ & $-0.08 \pm 0.1$ & 0.705 & -0.102 & 0.572 \\
\hline \multicolumn{6}{|l|}{ Muscle activity ${ }^{\mathrm{a}}$} \\
\hline Vastus medialis (VM) & $0.43 \pm 0.1$ & $0.41 \pm 0.1$ & 0.566 & 0.104 & 0.566 \\
\hline Vastus lateralis (VL) & $0.43 \pm 0.1$ & $0.43 \pm 0.1$ & 0.948 & 0.012 & 0.948 \\
\hline Medialis hamstrings (MH) & $0.36 \pm 0.1$ & $0.34 \pm 0.1$ & 0.507 & 0.120 & 0.507 \\
\hline Lateral hamstrings (LH) & $0.38 \pm 0.1$ & $0.38 \pm 0.1$ & 0.857 & 0.033 & 0.857 \\
\hline VMMH co-contraction & $0.60 \pm 0.2$ & $0.57 \pm 0.2$ & 0.683 & 0.074 & 0.683 \\
\hline VLLH co-contraction & $0.63 \pm 0.2$ & $0.65 \pm 0.2$ & 0.841 & -0.036 & 0.841 \\
\hline VLMH co-contraction & $0.60 \pm 0.2$ & $0.58 \pm 0.2$ & 0.834 & 0.038 & 0.834 \\
\hline VMMH/VLLH co-contraction ratio & $1.04 \pm 0.5$ & $0.96 \pm 0.4$ & 0.684 & 0.073 & 0.684 \\
\hline
\end{tabular}

Data are presented as mean \pm standard deviation and $r=$ Pearson correlation coefficient. No statistically significant differences between groups.

a Root mean square from touch-down to PKFA during the stepping-down task 
Knee muscle strength in OA patients with and without self-reported knee instability

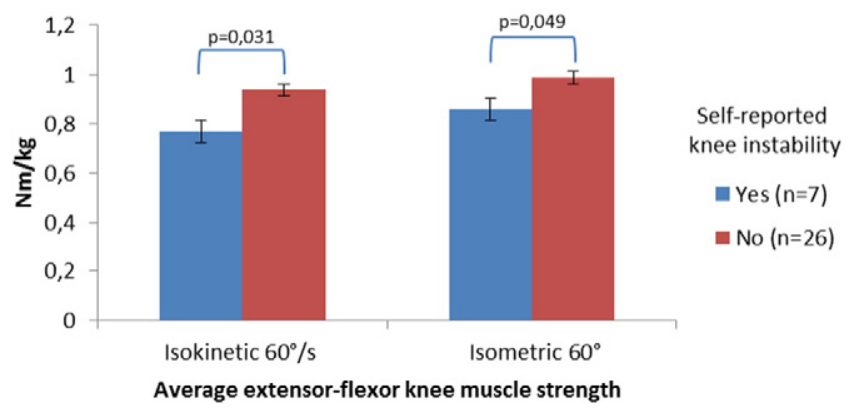

Figure 5. Distribution of knee muscle strength by self-reported knee instability.

daily living in patients with $\mathrm{OA}$. This might potentially contribute to slow down the joint damage and subsequent increase in activity limitations in this group of patients.

Several limitations of the present study should be considered. First, patients with uni- and bilateral knee OA were included in the study. It is possible that patients with bilateral knee OA might have developed different compensatory mechanisms to ambulate compared with patients who have only one knee affected. However, it is very likely that all patients might have had the contralateral knee (undiagnosed) affected to some extent. Overall, there is commonly well-accepted to use an index knee, which includes the more affected knee in patients with bilateral knee OA, for the analyses. Second, pain intensity during the performance of the stepping-down task was not assessed. Authors are aware that pain could have influenced the performance of the stepping-down task. Therefore, in a future study, gathering this information will be considered in order to adjust the analyses.

Third, only a small number of patients with knee OA reported a sensation of knee instability during the past three months. The small number of patients with this characteristic translated into a lack of statistical power which did not allow us to draw strong conclusions about the biomechanical characteristics in patients with self-reported knee instability from these analyses. Fourth, it was not possible to perform further analyses by frequency of knee instability also due to the small number of patients with self-reported knee instability. It is possible that patients with a higher number of episodes of knee instability may have different biomechanical characteristics than patients with a lower number of episodes. Therefore, self-reported knee instability should be used as an inclusion criterion on for further studies in order to evaluate the kinetic and kinematic characteristics associated with the sensation of knee instability. Fifth, differences in patients' height might have had a potential influence on descending from a step [54]. However, there were no statistical differences in height between the three study groups (Table 1). Additionally, a $20 \mathrm{~cm}$ step is considered a standard step height mimicking daily live scenarios involving stairs regardless of the height of the patients. Lastly, assessing a flight of stairs rather than one step-down might have revealed more differences.

\section{Conclusions}

The greater EMG activity found during the loading phase of the stepping-down task in established OA might suggest a less efficient use of knee muscles or an attempt to increase knee stability. Statistically significant differences in the other analysed variables were not found.

\section{Conflict of interest}

The authors of this article declare that they have no conflict of interest.

\section{Acknowledgements}

We would like to thank C. Smoulders and A. Mahmoudian for their assistance in gathering the data. This study was funded by the European Commission, through the Erasmus Mundus Joint Doctorate Move-age Program (2011-2015). The study sponsor had no involvement in the study.

\section{References}

[1] Zhang Y, Jordan JM. Epidemiology of osteoarthritis. Clin Geriatr Med 2010;26(3): 355-69.

[2] World Health Organization. The global burden of disease - 2004 update; 2008.

[3] Davis MA, Ettinger WH, Neuhaus JM, Mallon KP. Knee osteoarthritis and physical functioning: evidence from the NHANES I epidemiologic followup study. J Rheumatol 1991;18(4):591-8.

[4] World Health Organization. International classification of functioning, disability and health:ICF. Geneva: WHO; 2001.

[5] Astephen JL, Deluzio KJ. Changes in frontal plane dynamics and the loading response phase of the gait cycle are characteristic of severe knee osteoarthritis application of a multidimensional analysis technique. Clin Biomech (Bristol, Avon) 2005;20(2): 209-17.

[6] Chen CP, Chen MJ, Pei YC, Lew HL, Wong PY, Tang SF. Sagittal plane loading response during gait in different age groups and in people with knee osteoarthritis. Am J Phys Med Rehabil 2003;82(4):307-12.

[7] Baliunas AJ, Hurwitz DE, Ryals AB, Karrar A, Case JP, Block JA, et al. Increased knee joint loads during walking are present in subjects with knee osteoarthritis. Osteoarthritis Cartilage 2002;10(7):573-9.

[8] Childs JD, Sparto PJ, Fitzgerald GK, Bizzini M, Irrgang JJ. Alterations in lower extremity movement and muscle activation patterns in individuals with knee osteoarthritis. Clin Biomech (Bristol, Avon) 2004;19(1):44-9.

[9] Simic M, Hinman RS, Wrigley TV, Bennell KL, Hunt MA. Gait modification strategies for altering medial knee joint load: a systematic review. Arthritis Care Res (Hoboken) 2011;63(3):405-26.

[10] Lewek MD, Rudolph KS, Snyder-Mackler L. Control of frontal plane knee laxity during gait in patients with medial compartment knee osteoarthritis. Osteoarthritis Cartilage 2004;12(9):745-51.

[11] Thorp LE, Sumner DR, Block JA, Moisio KC, Shott S, Wimmer MA. Knee joint loading differs in individuals with mild compared with moderate medial knee osteoarthritis. Arthritis Rheum 2006;54(12):3842-9.

[12] Schmitt LC, Rudolph KS. Influences on knee movement strategies during walking in persons with medial knee osteoarthritis. Arthritis Rheum 8-15-2007;57(6): 1018-26.

[13] Heiden TL, Lloyd DG, Ackland TR. Knee joint kinematics, kinetics and muscle cocontraction in knee osteoarthritis patient gait. Clin Biomech (Bristol, Avon) 2009; 24(10):833-41.

[14] Hicks-Little CA, Peindl RD, Fehring TK, Odum SM, Hubbard TJ, Cordova ML. Temporal-spatial gait adaptations during stair ascent and descent in patients with knee osteoarthritis. J Arthroplasty 2012;27(6):1183-9.

[15] Guccione AA, Felson DT, Anderson JJ, Anthony JM, Zhang Y, Wilson PW, et al. The effects of specific medical conditions on the functional limitations of elders in the Framingham study. Am J Public Health 1994;84(3):351-8.

[16] Houck J, Lerner A, Gushue D, Yack HJ. Self-reported giving-way episode during a stepping-down task: case report of a subject with an ACL-deficient knee. J Orthop Sports Phys Ther 2003;33(5):273-82.

[17] Koyama Y, Tateuchi H, Nishimura R, Ji X, Umegaki H, Kobayashi M, et al. Relationships between performance and kinematic/kinetic variables of stair descent in patients with medial knee osteoarthritis: an evaluation of dynamic stability using an extrapolated center of mass. Clin Biomech (Bristol, Avon) 9-18-2015.

[18] Luyten FP, Denti M, Filardo G, Kon E, Engebretsen L. Definition and classification of early osteoarthritis of the knee. Knee Surg Sports Traumatol Arthrosc 2012;20(3): 401-6.

[19] van der Esch M, Knoop J, van der Leeden M, Voorneman R, Gerritsen M, Reiding D et al. Self-reported knee instability and activity limitations in patients with knee osteoarthritis: results of the Amsterdam osteoarthritis cohort. Clin Rheumatol 6-232012.

[20] Felson DT, Niu J, McClennan C, Sack B, Aliabadi P, Hunter DJ, et al. Knee buckling: prevalence, risk factors, and associated limitations in function. Ann Intern Med 10-16-2007;147(8):534-40.

[21] Knoop J, van der Leeden M, van der Esch M, Thorstensson CA, Gerritsen M, Voorneman RE, et al. Association of lower muscle strength with self-reported knee instability in osteoarthritis of the knee: results from the Amsterdam Osteoarthritis Cohort. Arthritis Care Res (Hoboken) 2012;64(1):38-45.

[22] Fisher NM, Pendergast DR. Reduced muscle function in patients with osteoarthritis Scand J Rehabil Med 1997;29(4):213-21.

[23] Sharma L, Lou C, Felson DT, Dunlop DD, Kirwan-Mellis G, Hayes KW, et al. Laxity in healthy and osteoarthritic knees. Arthritis Rheum 1999;42(5):861-70.

[24] Wada M, Imura S, Baba H, Shimada S. Knee laxity in patients with osteoarthritis and rheumatoid arthritis. Br J Rheumatol 1996;35(6):560-3.

[25] Lewek MD, Ramsey DK, Snyder-Mackler L, Rudolph KS. Knee stabilization in patients with medial compartment knee osteoarthritis. Arthritis Rheum 2005;52(9): 2845-53. 
[26] Sharma L, Dunlop DD, Cahue S, Song J, Hayes KW. Quadriceps strength and osteoarthritis progression in malaligned and lax knees. Ann Intern Med 4-15-2003;138(8): 613-9.

[27] Bennell KL, Hinman RS, Metcalf BR. Association of sensorimotor function with knee joint kinematics during locomotion in knee osteoarthritis. Am J Phys Med Rehabil 2004;83(6):455-63.

[28] Kaufman KR, Hughes C, Morrey BF, Morrey M, An KN. Gait characteristics of patients with knee osteoarthritis. J Biomech 2001:34(7):907-15.

[29] Hortobagyi T, DeVita P. Altered movement strategy increases lower extremity stiffness during stepping down in the aged. J Gerontol A Biol Sci Med Sci 1999;54(2): B63-70.

[30] Houck J, Yack HJ. Giving way event during a combined stepping and crossover cutting task in an individual with anterior cruciate ligament deficiency. J Orthop Sports Phys Ther 2001;31(9):481-9.

[31] Altman R, Asch E, Bloch D, Bole G, Borenstein D, Brandt K, et al. Development of criteria for the classification and reporting of osteoarthritis. Classification of osteoarthritis of the knee. Diagnostic and Therapeutic Criteria Committee of the American Rheumatism Association. Arthritis Rheum 1986;29(8):1039-49.

[32] Kellgren JH, Lawrence JS. Radiological assessment of osteo-arthrosis. Ann Rheum Dis 1957;16:494-502.

[33] Felson DT, Niu J, Guermazi A, Sack B, Aliabadi P. Defining radiographic incidence and progression of knee osteoarthritis: suggested modifications of the Kellgren and Lawrence scale. Ann Rheum Dis 2011;70(11):1884-6.

[34] Sanchez-Ramirez DC, van der Leeden M, van der Esch M, Roorda LD, Verschueren S, van Dieen J, et al. Increase in knee muscle strength is associated with a decrease in activity limitations in established knee osteoarthritis: a 2 year follow-up study in the Amsterdam Osteoarthritis (AMS_OA) cohort. J Rehabil Med 2015 (accepted for publication).

[35] Baert IA, Mahmoudian A, Nieuwenhuys A, Jonkers I, Staes F, Luyten FP, et al. Proprioceptive accuracy in women with early and established knee osteoarthritis and its relation to functional ability, postural control, and muscle strength. Clin Rheumatol 2013;32(9):1365-74

[36] Kean CO, Birmingham TB, Garland SJ, Bryant DM, Giffin JR. Minimal detectable change in quadriceps strength and voluntary muscle activation in patients with knee osteoarthritis. Arch Phys Med Rehabil 2010;91(9):1447-51.

[37] Baert IA, Staes F, Truijen S, Mahmoudian A, Noppe N, Vanderschueren G, et al. Weak associations between structural changes on MRI and symptoms, function and muscle strength in relation to knee osteoarthritis. Knee Surg Sports Traumatol Arthrosc 2-2-2013.

[38] Cooke TD, Sled EA, Scudamore RA. Frontal plane knee alignment: a call for standardized measurement. J Rheumatol 2007;34(9):1796-801.

[39] de Groot IB, Favejee MM, Reijman M, Verhaar JA, Terwee CB. The Dutch version of the knee injury and osteoarthritis outcome score: a validation study. Health Qual Life Outcomes 2008;6:16.

[40] Malfait B, Sankey S, Firhad Raja Azidin RM, Deschamps K, Vanrenterghem J, Robinson MA et al. How reliable are lower-limb kinematics and kinetics during drop vertical jump? Med Sci Sports Exerc 2014;46(4):678-85.

[41] Besier TF, Sturnieks DL, Alderson JA, Lloyd DG. Repeatability of gait data using a functional hip joint centre and a mean helical knee axis. J Biomech 2003;36(8):1159-68.
[42] Vanrenterghem J, Venables E, Pataky T, Robinson MA. The effect of running speed on knee mechanical loading in females during side cutting. J Biomech 9-21-2012; 45(14):2444-9.

[43] Hermens HJ, Freriks B, Disselhorst-Klug C, Rau G. Development of recommendations for SEMG sensors and sensor placement procedures. J Electromyogr Kinesiol 2000; 10(5):361-74

[44] Kendall FP, Kendall McCreary E, Provance PG. Muscle-testing and function. 4th ed. Baltimore: Williams and Wilkins; 1993.

[45] Winter DA, Fuglevand AJ, Archer SE. Crosstalk in surface electromyography: theoretical and practical estimates. J Electromyogr Kinesiol 1994;4(1):15-26.

[46] Cappozzo A, Catani F, Croce UD, Leardini A. Position and orientation in space of bones during movement: anatomical frame definition and determination. Clin Biomech (Bristol, Avon) 1995;10(4):171-8.

[47] Schwartz MH, Rozumalski A. A new method for estimating joint parameters from motion data. J Biomech 2005;38(1):107-16.

[48] Bisseling RW, Hof AL. Handling of impact forces in inverse dynamics. J Biomech 2006;39(13):2438-44.

[49] Baert IA, Jonkers I, Staes F, Luyten FP, Truijen S, Verschueren SM. Gait characteristics and lower limb muscle strength in women with early and established knee osteoarthritis. Clin Biomech (Bristol, Avon) 2013;28(1):40-7.

[50] Dempster WT, Gaughran GR. Properties of body segments based on size and weight. Am J Anat 1967:120(1):33-54.

[51] Robertson DG, Dowling JJ. Design and responses of Butterworth and critically damped digital filters. J Electromyogr Kinesiol 2003;13(6):569-73.

[52] Yang JF, Winter DA. Electromyographic amplitude normalization methods: improving their sensitivity as diagnostic tools in gait analysis. Arch Phys Med Rehabil 1984; 65(9):517-21.

[53] Saywell N, Taylor D, Boocock M. During step descent, older adults exhibit decreased knee range of motion and increased vastus lateralis muscle activity. Gait Posture 2012;36(3):490-4

[54] Rudolph KS, Axe MJ, Snyder-Mackler L. Dynamic stability after ACL injury: who can hop? Knee Surg Sports Traumatol Arthrosc 2000;8(5):262-9.

[55] Palmieri-Smith RM, McLean SG, Ashton-Miller JA, Wojtys EM. Association of quadriceps and hamstrings cocontraction patterns with knee joint loading. J Athl Train 2009;44(3):256-63.

[56] Schmitt LC, Fitzgerald GK, Reisman AS, Rudolph KS. Instability, laxity, and physical function in patients with medial knee osteoarthritis. Phys Ther 2008;88(12): $1506-16$.

[57] Messier SP, DeVita P, Cowan RE, Seay J, Young HC, Marsh AP. Do older adults with knee osteoarthritis place greater loads on the knee during gait? A preliminary study. Arch Phys Med Rehabil 2005;86(4):703-9.

[58] Fitzgerald GK, Childs JD, Ridge TM, Irrgang JJ. Agility and perturbation training for a physically active individual with knee osteoarthritis. Phys Ther 2002;82(4):372-82.

[59] Al-Khlaifat L, Herrington L, Hammond A, Jones RK. Effect of a six-week combined hip and knee neuromuscular exercise programme on joint loading and muscle cocontraction in individuals with medial knee osteoarthritis. Osteoarthritis Cartilage 2013;21(Supplement):S98. 